\title{
IL VERBO ITALIANO DI MODO FINITO NELLA STRUTTURA INFORMATIVA DELL'ENUNCIATO A CONFRONTO CON IL VERBO INGLESE
}

\author{
Eva Klímová
}

\begin{abstract}
The article deals with the Italian and the English finite verb form from the Functional Sentence Perspective (FSP) point of view. The morphological and syntactical differences between both languages present Italian and English as two typologically different languages. These differences have their reflection on the FSP level. From this point of view the Italian and the English finite verb forms are observed as to their position in the sentence and within the communicative units.
\end{abstract}

Key words: Italian and English finite verb form; Functional Sentence Perspective; position; communicative units.

Riassunto: Nell' articolo il verbo a modo finite in italiano e in inglese vengono osservati dal punto di vista della struttura informativa, ovvero prospettiva funzionale dell'enunciato (PFE). Le differenze morfologiche e sintattiche tra le due lingue in questione presentano l'italiano e inglese come due lingue tipologicamente diverse. Le differenze tipologiche si riflettono a livello della prospettiva funzionale dell'enunciato. Da questo punto di vista il verbo italiano e messo a confronto con il verbo inglese osservando la posizione che essi occupano nella frase e nell' ambito delle unità comunicative.

Parole chiave: verbo di modo finito italiano e inglese; struttura informativa; posizione; unità comunicative.

\section{Introduzione}

Il verbo predicativo viene abitualmente indicato come centro della frase almeno per due livelli: al livello sintattico la funzione centrale del verbo predicativo deriva dal fatto che da questo viene compiuto, per mezzo delle categorie predicative di tempo e di modo, l'atto predicativo, ovvero l'atto della costituzione della frase. Al livello semantico, il ruolo centrale del verbo predicativo è legato alle sue valenze; infatti è il significato del verbo predicativo a determinare il numero e il tipo dei suoi argomenti. Determina, cioè, la struttura sintattica della frase. Lo scopo dell'articolo è quello di dimostrare la centralità della funzione del verbo predicativo anche per un terzo livello: quello della struttura informativa, ovvero della prospettiva funzionale dell' enunciato (PFE). A questo proposito il verbo predicativo verrà osservato da alcuni punti di vista: rispetto alla sua posizione nei confronti degli altri costituenti della frase e rispetto alla funzione degli 
elementi grammaticali e non grammaticali in cui il verbo è scomponibile. Per poterlo fare riteniamo indispensabile introdurre i termini e concetti di base di cui avvalerci in seguito:

- La teoria della PFE considera l'enunciato come campo comunicativo e i suoi elementi come distinte unità comunicative.

- Le unità comunicative in questione sono: tema, transizione propria, transizione e rema. ${ }^{1}$ A ciascuna unità comunicativa viene attribuito un certo grado di dinamismo comunicativo (DC), cioè «la misura relativa in cui un elemento contribuisce allo sviluppo ulteriore della comunicazione». (Firbas 1991: 198)

- Il grado di DC risulta dall'interazione dei quattro fattori della PFE, cioè ordinamento lineare, struttura semantica, contesto e, nel parlato, intonazione. ${ }^{2}$ Questi fattori operano in varie lingue con vigore diverso, tuttavia sempre e immancabilmente in interazione tra di loro. Se, per esempio, per l'italiano viene constatata una certa libertà nell'ordine delle parole, il grado di DC del costituente rispettivo, oltre che dalla funzione semantica, può essere segnalato dalla posizione nella frase. In altre parole, un costituente può essere confermato nella sua funzione tematica o rematica non solo dal suo carattere di "noto" o "nuovo" ma anche dalla sua posizione nella frase.

- Per tema s'intende quell'elemento dell'enunciato che porta il grado di DC più basso, per rema, invece s'intende l'elemento che ne porta il grado massimo.

La funzione del verbo italiano di modo finito e la funzione di quello inglese riflettono differenze osservabili a livello morfologico e sintattico essendo l'italiano, grazie alla ricchezza della flessione verbale, una lingua tipicamente sintetica, ed essendo l'inglese, caratterizzato da una scarsa flessione verbale, una lingua tipicamente analitica.

Sono appunto le differenze tra le lingue in questione nella flessione verbale che permettono di valutare il verbo in base alla sua posizione, tenendo, però, in mente che l'ordinamento lineare è solo uno dei fattori della struttura informativa dell'enunciato. La funzione del verbo viene cioè influenzata, se non determinata, dalla funzione semantica che esso svolge nelle scale semantiche di Firbas (vedi più avanti i loro schemi). Quindi il verbo predicativo viene osservato rispetto alla sua funzione nell'ambito delle unità comunicative elencate sopra. L'esempio che segue serve ad illustrare il nostro approccio: ${ }^{4}$

(1) ... e nelle vetrine delle drogherie si imbiancavano biscotti ammuffiti. GEN p. 39 ..., and in the windows of grocers' shops musty biscuits lay bleaching. DUB p. 15

La versione italiana e quella inglese si differenziano, a prima vista, solo per quanto riguarda l'ordine dei costituenti, cioè l'ordinamento lineare: il soggetto biscotti ammuffiti/ musty biscuits rappresenta un elemento non ricavabile dal contesto e, quindi, svolge la funzione di rema. Nella versione italiana esso segue il verbo, mentre nella versione inglese lo precede. Ciò vuol dire che nella prospettiva di quest'enunciato italiano è stato impiegato, oltre al contesto, l'ordinamento lineare, mentre nell'enunciato inglese è stata, oltre al contesto, la struttura semantica ad esprimere la prospettiva. Per la versione ita-

\footnotetext{
1 Le unità comunicative di tema e di rema sono ulteriormente scomponibili in tema proprio, tema tendente al tema proprio, tema tendente al diatema, diatema, rema e, rema proprio. Cfr. Svoboda 1991, p. 432.

2 Cfr. Firbas 1991, p. 197

3 Cfr. Firbas 1966, p. 240.

4 Per le fonti degli esempi e per le abbreviazioni vedi la lista dei testi che segue la bibliografia. I numeri indicano la pagina della fonte. Per l'analisi dell'esempio 1 cfr. Klímová 2007a.
} 
liana si può, quindi, constatare la cosiddetta distribuzione fondamentale del $D C$, mentre per la versione inglese no.

Altre differenze tra il verbo italiano e quello inglese sono osservabili nella loro struttura e nella forma: nella struttura del verbo italiano si imbiancavano sono identificali il prefisso im- e il suffisso -are per mezzo dei quali è stato derivato dall'aggettivo bianco il verbo significante diventare bianco. Sintatticamente esso rappresenta un predicato sintetico, semanticamente esso esprime sia il significato di bianco che il significato del cambiamento dello stato, cioè da non essere bianco ad esserlo. Il significato del verbo inglese lay, rispetto a quello italiano, è più vago. Non esprime altro che lo stato. Morfologicamente, la forma verbale italiana esprime le categorie grammaticali di persona, di numero, di tempo grammaticale e di modo. Con la forma dell'imperfetto l'azione è indicata come incompiuta, mentre la forma del verbo inglese esprime le categorie grammaticali di tempo e modo, le categorie di persona e numero, invece, sono riportate esclusivamente dal soggetto della frase. In più, il preterito inglese è indifferente rispetto alla categoria dell' aspetto. Esso, assieme al significato diventare bianco, è espresso dalla forma non-finita bleaching. Tutte queste differenze morfosintattiche e semantiche si riflettono con molta chiarezza nella funzione comunicativa del verbo finito nelle lingue in questione, cioè nel ruolo che esso svolge nella struttura informativa dell'enunciato.

\section{Posizione del verbo finito nella frase}

Per poter stabilire la funzione del verbo predicativo nella struttura informativa dell'enunciato, bisogna definire la sua posizione rispetto agli altri costituenti della frase. Mettendo a confronto l'italiano e l'inglese si constata, per l'italiano, una notevole libertà nell' ordinamento lineare. Di conseguenza si ottiene facilmente la cosiddetta distribuzione fondamentale del DC (come dimostra sopra l'esempio 1). L'inglese, invece, viene definito come lingua dall'ordine piuttosto fisso, grammaticalizzato, in cui la posizione dei costituenti è legata alla loro funzione sintattica. Di conseguenza il grado di DC viene ad essi attribuito in dipendenza del contesto. Tuttavia, per tutte e due le lingue, prive del caso morfologico dei nomi, viene indicato come non marcato l'ordine $(S)$ oggetto- $(V)$ erbo- $(O)$ ggetto che, in italiano, è modificabile grazie alla flessione del verbo e del pronome personale. Le possibilità di modificare l'ordine grammaticalizzato in inglese sono estremamente limitate. La scarsa flessione del verbo permette di identificarlo quasi esclusivamente per la sua posizione nella frase (cfr. per esempio work hard e hard work). Per dimostrare le differenze tra l'ordinamento lineare in italiano e in inglese riteniamo opportuno osservare la posizione e funzione del verbo predicativo negli schemi delle scale semantiche. ${ }^{5}$ Successivamente il verbo verrà descritto sulla base della sua posizione rispetto agli argomenti e circostanziali.

\subsection{Verbo nella scala presentativa}

Nella scala presentativa con lo schema scena $\rightarrow$ esistenza/apparizione sulla scena $\rightarrow$ fenomeno (sulla scena), il verbo finito, indipendentemente dalla sua posizione, ha la funzione semantica di esistenza/apparizione sulla scena e, rispetto alle unità comunicative, svolge la funzione di transizione. Il soggetto grammaticale della frase ha la funzione semantica di fenomeno e, per quanto riguarda le unità comunicative, è il rema dell'enunciato. In altre

\footnotetext{
5 Cfr. Klímová 2004.
} 
parole, nella scala presentativa la comunicazione è orientata verso il soggetto grammaticale. Vediamo l'esempio:

$\begin{array}{ll}\text { (2) Entrò Carla. } & \text { IND p. } 1 \\ \text { Carla came into the room. } & \text { TIM p. } 5\end{array}$

Con questa frase si introduce "sulla scena" Carla, uno dei protagonisti del romanzo Gli indifferenti di A. Moravia. ${ }^{6}$ Trattandosi della prima frase del romanzo, non ha nessun contesto linguistico. Nella versione italiana, il soggetto/fenomeno Carla occupa la posizione postverbale, mentre nella versione inglese tale elemento viene osservato prima del verbo. Nella versione italiana, oltre alla funzione semantica, è l'ordinamento lineare a segnalare il grado di DC degli elementi della frase, mentre nella versione inglese è solo la loro funzione semantica a indicarlo. (Cfr. inoltre l'esempio 1 nella parte introduttiva)

\subsection{Verbo nella scala di qualità}

Nella scala di qualità con lo schema (scena) $\rightarrow$ portatore (della qualità) $\rightarrow$ qualità $\rightarrow$ specificazione ( $\rightarrow$ altra specificazione) il verbo finito ha la funzione semantica di qualità ed è, rispetto alle unità comunicative nel caso di assenza della specificazione, il rema; nel caso di presenza della specificazione, è la transizione dell'enunciato. In altre parole, nella scala di qualità la comunicazione è orientata verso un elemento diverso dal soggetto grammaticale. Confrontiamo gli esempi successivi:

(3) Alla porta si fermarono. IND p. 14

At the door they stopped. TIM p. 14

Il costituente alla porta/at the door in posizione iniziale fa parte del contesto situazionale e, fungendo da scena, è un elemento tematico. Fa parte del tema anche il soggetto/portatore della qualità. Il verbo si fermarono/stopped è la qualità che svolge la funzione rematica.

(4) Nel corridoio incontrò Michele,... IND p. 12

In the corridor she met Michele TIM p. 12

Il costituente nel corridoio/in the corridor funge da scena ed è tematico. (Anche qui il soggetto grammaticale fa parte del tema) Il verbo incontrò/met è la qualità e funge da transizione.

\subsection{Posizione del verbo rispetto al soggetto}

La flessione del verbo italiano ne facilita l'identificazione nella frase e, accanto all'ordinamento $S-V$ nel caso del soggetto ancorato nel contesto, come nell'esempio 5, rende possibile l'ordinamento $V-S$ nel caso del soggetto non ricavabile dal contesto, come nell'esempio 6:

(5) E la madre usci.

IND p. 14

And Michele's mother went out. TIM p. 14

(6) Segui un minuto di silenzio. IND p. 19

There was a moment of silence. TIM p. 18

Ricordiamo che la posizione del verbo è legata alla sua funzione semantica: seguendo il soggetto funge da qualità, precedendolo esprime esistenza/apparizione sulla scena. In inglese, l'ordinamento $V$-S è possibile solo grazie alla struttura di esistenza there is/ are corrispondente alla struttura italiana $c^{\prime} e ̀ / c i$ sono, come dimostra l'esempio successivo:

\footnotetext{
6 Cfr. la frase modello A boy came into the room in Firbas 1992, p. 59.
} 
Il verbo italiano di modo finito nella struttura informativa dell'enunciato...

$\begin{array}{ll}\text { (7) C'erano delle macchie, il vino. } & \text { IND p. } 17 \\ & \text { There were stains upon it - the red of wine. }\end{array}$

\subsection{Posizione del verbo rispetto al complemento oggetto ed al complemento di termine}

Esaminando la posizione del verbo italiano e quello inglese rispetto al complemento oggetto ed al complemento di termine, non si osservano differenze lì dove questi complementi svolgono la funzione di rema. In quel caso l'ordinamento è $S-V-O$ in tutte e due le lingue. Invece, lì dove il complemento rappresenta un elemento tematico, soprattutto nell'italiano parlato, si incontrano le strutture chiamate dislocazioni. In queste strutture un elemento tematico viene "dislocato" e pronominalizzato. La ripresa pronominale ne indica univocamente la funzione sintattica:

(8) Io la mano la bacio soltanto alle donne. $\quad$ GATT p. 209 Usually I kiss only ladies' hands. $\quad$ LEOP p. 160

Nella versione italiana di questo esempio si osserva la dislocazione a sinistra del complemento oggetto la mano, successivamente pronominalizzato con il clitico la. Occupando la posizione iniziale, l'elemento dislocato è esplicitamente indicato come tematico (assieme al soggetto io). A fungere da rema è il complemento di termine alle donne. In inglese, sarebbe l'intonazione a indicare il costituente ladies' come rema. Nell'esempio (9) viene esemplificata la dislocazione a destra:

(9) (... non solamente gli faccio le scuse) ma gli bacio anche i piedi $a$ Leo. IND p. 35

( ... I'll not only apologize to Leo) but kiss his feet too.

TIM p. 33

Come si vede, il complemento di termine $a$ Leo, che deve essere indicato come tematico, viene anticipato con la ripresa pronominale gli. In inglese, il complemento di termine to Leo occupa la posizione grammaticalizzata e, anche in questo caso, la struttura informativa dell'enunciato è indicata dal contesto e dalla intonazione.

Con le dislocazioni l'italiano ha uno strumento molto efficace per indicare un costituente della frase. Fanno sì che nella frase italiana si ottenga la distribuzione fondamentale del DC.

\subsection{Posizione del verbo rispetto ai complementi di luogo e di tempo}

Per la posizione dei complementi di luogo e di tempo, in italiano, il fattore decisivo è la loro funzione semantica nelle scale i cui schemi sono stati descritti sopra: in funzione della scena, locale o temporale, appartengono al tema occupando la posizione iniziale nella frase, cioè prima del verbo; in funzione della specificazione o specificazione ulteriore sono rematici, in posizione finale, dopo il verbo. In inglese, anche la posizione degli elementi avverbiali è determinata dalle regole grammaticali, anche se pure lì si osservano tendenze a posizionarli sulla base della loro funzione semantica. Vediamo la frase già usata nell'esempio 3 sopra:

(10) Alla porta si fermarono. IND p. 14 At the door they stopped. TIM p. 14

L'avverbiale alla porta/at the door in posizione iniziale è la scena e funge da tema. Il verbo in posizione finale è rematico. Nella frase

$\begin{array}{lll}\text { (11) E nei giorni normali nella vetrina era appeso un avviso che ... } & \text { GEN p. } 29 \\ \text { And on ordinary days a notice used to hang in the window, saying... } & \text { DUB p. } 3\end{array}$ 
gli avverbiali nei giorni normali e nella vetrina sono scena e occupano la posizione iniziale, in corrispondenza con la loro funzione semantica; il soggetto un avviso è un fenomeno nella scena e in funzione di rema occupa la posizione dopo il verbo. Nella versione inglese il complemento di tempo on ordinary days viene osservato in posizione iniziale, prima del verbo; il complemento di luogo in the window, invece, in posizione finale, dopo il verbo. Il soggetto a notice occupa la posizione preverbale, così come richiede la regola grammaticale. Si può quindi concludere che anche in questo caso nell'enunciato italiano, al contrario di quello inglese, si ottiene la distribuzione fondamentale del DC.

\section{La funzione del verbo nelle unità comunicative}

Per poter valutare le funzioni del verbo predicativo nell' ambito delle unità comunicative elencate sopra, bisogna prendere in considerazione il fatto che nella struttura del verbo finito vanno distinti le categorie grammaticali e il significato lessicale. Le categorie di numero e di persona rappresentano l'esponente personale e numerale (EPN), le categorie grammaticali di tempo e di modo rappresentano l'esponente temporale e modale (ETM); il significato lessicale è la componente nozionale. ${ }^{7}$ Esaminando gli elementi grammaticali e non grammaticali si può scoprire sia il valore comunicativo sia il suo grado di DC: in italiano, il verbo di modo finito, esprimendo tutte le categorie grammaticali, può partecipare ad alcune unità comunicative e, con la componente nozionale, fungere da rema.

\subsection{Esponente personale e numerale del verbo}

Una delle differenze più significative concernenti l'EPN sta nel fatto che il verbo italiano di modo finito esprime le categorie di persona e di numero, indicando così univocamente il soggetto della frase. Il verbo inglese, invece, non lo indica, richiedendo esso la presenza del soggetto pronominale. Questa differenza si manifesta nella funzione delle unità comunicative: ${ }^{8}$

(12) Spesso quando ci pensavo ...

GEN p. 30

Often when I thought of this ... $\quad$ DUB p. 5

La forma del verbo italiano pensavo indica il soggetto; quindi l'EPN del verbo, assieme al clitico $c i$, partecipa al tema. La forma del verbo inglese thought è indifferente rispetto al numero e alla persona, cioè non contiene l'EPN. La funzione di tema, oltre che dalla struttura preposizionale of this, è svolta dal soggetto pronominale I. Così si può constatare che il valore comunicativo del verbo italiano è più alto di quello del verbo inglese.

\subsection{Esponente temporale e modale}

L'esponente temporale e modale svolge, costantemente, la funzione dell'unità comunicativa di transizione propria. ${ }^{9}$ L'esponente modale verrà esaminato dopo, separatamente dall'esponente temporale.

\subsubsection{Esponente modale}

Esaminando l'esponente modale (EM), bisogna, prima di tutto, dedicarsi alla base modale dell'enunciato, costituita dal modo verbale, che sta nel centro della transizione propria

\footnotetext{
Cfr. Firbas 1992, p. 70.

8 Cfr. Klímová 2007a.

9 Cfr. Svoboda 1989 , p. 66.
} 
Il verbo italiano di modo finito nella struttura informativa dell'enunciato...

$\left(\mathrm{TR}_{\text {prop }}\right)$. Accanto al centro, però, appaiono significati modali della modalità deontica ed epistemica che sono osservabili nella sua periferia (periferia ${ }^{+}$e periferia-).

In italiano, come anche in inglese, ci sono quattro modi verbali finiti: indicativo, imperativo, congiuntivo e condizionale. La differenza tra queste due lingue, per quanto riguarda il modo, sta prima di tutto nel fatto che il congiuntivo italiano è sempre uno strumento vivo e insostituibile, mentre in inglese, morfologicamente, il congiuntivo è difficilmente identificabile, il che ha condotto al suo disuso. Vediamo l'EM nello schema dei tipi di frase:

- L'EM della frase dichiarativa e di quella interrogativa corrisponde all'indicativo e al condizionale:

$\begin{array}{ll}\text { (13) } & \text { Si preparano grandi cose, ... } \\ \text { Great things are in the offing and ... } & \text { GATT p. } 40 \\ \text { LEOP p. } 21\end{array}$

- L'EM della frase iussiva in italiano è l'imperativo, con la funzione del cosiddetto imperativo di cortesia viene usato il congiuntivo (quando si dà del lei). In inglese funge da imperativo la forma verbale di base, la forma per un imperativo di cortesia non esiste, perciò si fanno strada diverse strutture con verbi modali. Questa differenza si riflette nella struttura della $\mathrm{TR}_{\text {prop }}$ :

(14) Lei ci conceda questa proroga, ... IND p. 26

You must give us this respite. TIM p. 24

Il congiuntivo italiano conceda, in questa frase, funge da "imperativo di cortesia". La forma verbale inglese give con la funzione di imperativo non è sufficiente a rendere lo stesso significato. Il traduttore si è avvalso all'uso del verbo modale must che esprime esplicitamente la necessità deontica. Questa differenza tra il predicato della versione italiana e quello di quella inglese è osservabile nella struttura della $\mathrm{TR}_{\text {prop }}$ : mentre in italiano è un modo verbale, il congiuntivo, a esprimere l'EM nel centro della $\mathrm{TR}_{\text {prop, }}$ in inglese il significato modale di necessità si realizza come un elemento nella periferia ${ }^{+}$di quest' unità comunicativa (cfr. più avanti es. 17).

- Come EM della frase per le ottative funge, in italiano, il congiuntivo, indistinguibile, in inglese, dalla forma del preterito:

(15) (Se tu) sapessi. IND p. 7 If you only knew. TIM p. 6

Come dimostra l'esempio, al congiuntivo imperfetto italiano corrisponde la struttura perifrastica inglese "if only + preterito" (considerabile, però, anche la forma del congiuntivo). In altre parole, lì dove la forma verbale italiana è del tutto autosufficiente nel rendere il significato modale nel centro della $\mathrm{TR}_{\text {prop }}$ la forma inglese non lo è. Quindi si può constatare, anche in questo caso, che il valore comunicativo del verbo italiano è più alto di quello del verbo inglese.

- Da elemento nella periferia- della $\mathrm{TR}_{\text {prop }}$ fungono i significati della modalità epistemica espressi dagli strumenti lessicali, cioè da avverbi, come dimostra l'esempio successivo:

(16) Sono forse più intelligente, sono certamente più colto di ... $\quad$ GATT p. 202 I may be more intelligent, I' $m$ certainly more cultivated than ... LEOP p. 154

- Da elemento nella periferia ${ }^{+} \mathrm{TR}_{\text {prop }}$ fungono i significati della modalità deontica legati all'uso dei verbi modali, espressi cioè da strumenti lessicali:

(17) Ma i Re che incarnano un'idea non possono, non devono scendere per generazioni al di sotto di un certo livello. GATT p. 28 
But kings who personify an idea should not, cannot fall below a certain level for generations LEOP p. 10

Nell'esempio si vede il verbo modale potere in italiano e il verbo modale inglese should, che hanno il significato della possibilità deontica, e il verbo modale dovere e il verbo modale inglese cannot con il significato della necessità deontica.

- I significati modali, sia quelli centrali sia quelli periferici nell'ambito della TR ${ }_{\text {prop' }}$ sono esprimibili schematicamente così:

$\begin{array}{ccccc}\text { ME } & \leftrightarrow & \text { Base modale } & \leftrightarrow & \text { MD } \\ \downarrow & & \downarrow & & \downarrow \\ \text { Periferia }^{-} & \leftrightarrow & \text { Centro della TR }_{\text {prop }} & \leftrightarrow & \text { Periferia }^{+}\end{array}$

\subsubsection{Esponente temporale}

L'esponente temporale (ET), rappresentato dai tempi che mettono in relazione l'azione espressa dal verbo predicativo e il momento dell'enunciazione, è, come l'EM, una componente nel centro della $\mathbf{T R}_{\text {prop }}$. Da questo punto di vista non c'è nessuna differenza tra l'italiano e l'inglese:

(18) Tutti ci guardavano.

IND p. 6

Everyone was looking at us.

TIM p. 7

Con l'imperfetto italiano guardavano, corrispondente alla forma inglese was looking, l'azione viene indicata come anteriore rispetto al momento dell'enunciazione. Allo stesso tempo tutte e due le forme esprimono imperfettività. Il significato di anteriorità rappresenta l'ET nel centro della $\mathrm{TR}_{\text {prop' }}$ mentre il significato di imperfettività è una componente nella sua periferia. Vediamo ancora un esempio:

(19) ..., al che sorrideva e accennava una o due volte con la testa. ... upon which he used to smile and nod his head twice or thrice.

GEN p. 30

DUB p. 5

Le forme dell'imperfetto italiano sorrideva e accennava corrispondono alle forme perifrastiche inglesi used to smile e (used to) nod. Assieme all'anteriorità l'imperfetto italiano esprime "abitualità". Accanto all'ET nel centro della TR ${ }_{\text {prop }}$ è osservabile una componente nella periferia di essa.

Dopo aver paragonato il sistema dei tempi in italiano e in inglese, che rappresentano l'ET della $\mathrm{TR}_{\text {prop' }}$ siamo arrivati a una lista dei significati temporali nella sua periferia ${ }^{+}$ e periferia- Nella periferia ${ }^{+}$si osserva aspetto (perfettivo e imperfettivo), fase dell'azione, significato "inclusivo" e quello di "raggiungimento della meta dell'azione, significato "risultativo" e significato di "esperienza". Nella periferia- si distinguono "attualità", aspetto iterativo, "simultaneità" e "abitudine". Le potenzialità dei sistemi temporali in italiano e in inglese non sono uguali. Mentre il sistema dei tempi in italiano è perfettamente autosufficiente per esprimere tutti i significati elencati sopra, il sistema dei tempi in inglese non lo è. ${ }^{10}$

- Come i significati modali, anche quelli temporali distinguibili nel centro e nella periferia della $\mathrm{TR}_{\text {prop }}$ sono esprimibili schematicamente:

\footnotetext{
${ }_{10}$ Per i significati temporali periferici dei tempi in italiano e in inglese e per altri esempi cfr. Klímová 2003.
} 
Il verbo italiano di modo finito nella struttura informativa dell'enunciato...

\begin{tabular}{ccccc}
\hline Quantificatori temporali & $\leftrightarrow$ & Sistema dei tempi & $\leftrightarrow$ & Aspetto e fase \\
$\downarrow$ & & $\downarrow$ & & \\
Periferia $^{-}$ & $\leftrightarrow$ & Centro della TR & $\leftrightarrow$ &
\end{tabular}

Anche in questo caso bisogna constatare che lì dove il sistema dei tempi in inglese non è sufficiente per indicare alcuni significati temporali, il valore comunicativo del verbo italiano è più alto di quello del verbo inglese.

\subsection{Componente nozionale del verbo}

La componente nozionale del verbo viene osservata separatamente dai suoi EPN, EM e ET. Ricordiamo solo che essa nella scala presentativa è sempre transizione, nella scala di qualità può fungere o come transizione (nel caso di presenza di specificazione), o come rema (nel caso dell'assenza di specificazione). In altre parole, se nell'enunciato è presente un elemento che con il suo grado di DC supera la componente nozionale del verbo, quest'ultimo funge da transizione. Invece, se nell'enunciato un elemento del genere non è presente, la componente nozionale del verbo è il rema (cfr. esempi 3 e 4). Il grado di DC della componente nozionale del verbo è legato alla struttura del predicato:

(20) È pallido.

$$
\text { He is pale. }
$$

La componente nozionale del verbo copulativo è pari a zero, e quindi il verbo, riportando solo l'ETM, funge da TR prop. L'aggettivo del predicato pallido/pale esprime lo stato e funge da rema.

La situazione è più evidente lì dove l'italiano si differenzia dall'inglese per le sue capacità di derivare verbi il cui significato comprende più elementi semantici (cfr. es 1). Grazie a queste potenzialità derivative si osserva, in italiano, rispetto all'inglese, una forte tendenza alla sinteticità:
(21) Impallidi.
IND p. 26
She turned pale.
TIM p. 25

Il verbo italiano impallidire, che è stato creato, con il prefisso im- e il suffisso -ire, dall'aggettivo pallido, esprime due significati: quello del cambiamento e quello del risultato del cambiamento. Sintatticamente, esso rappresenta un predicato sintetico. Nel costrutto inglese she turned pale i due significati sono separati, sicché costituiscono un predicato analitico: il verbo turn esprime il significato del cambiamento, mentre l'aggettivo pale il significato del risultato del cambiamento. La differenza sintattica tra il predicato sintetico e quello analitico si riflette sulla funzione della componente nozionale del verbo predicativo: la forma verbale impallidì, oltre all'EPN del tema e all'ETM di TR ${ }_{\text {prop }^{\prime}}$ comprende la componente nozionale che funge da rema. La forma verbale inglese she turned, dopo la separazione dell'ETM, contiene la componente nozionale di cambiamento. Essa funge da transizione; a fungere da rema è l'aggettivo pale (l'EPN viene reso dal soggetto pronominale she). Così il verbo predicativo italiano supera quello inglese non solo per il valore comunicativo ma anche per il grado di DC della sua componente nozionale. ${ }^{11}$

\footnotetext{
11 Cfr. Klímová 2006.
} 


\section{Conclusioni}

Nel saggio il verbo predicativo italiano è stato messo a confronto con il verbo inglese dal punto di vista della prospettiva funzionale dell'enunciato. A questo proposito esso è stato esaminato per quanto riguarda la sua posizione nei confronti degli altri costituenti della frase e rispetto alla funzione degli elementi grammaticali e non grammaticali in cui il verbo è scomponibile, con lo scopo di dimostrare la sua centralità nella frase anche al livello della struttura informativa. In base all' analisi degli esempi nonché delle osservazioni cui si è fatto riferimento arriviamo alle seguenti conclusioni: a) il verbo di modo finito italiano, grazie alla sua flessione, a confronto con la ridotta flessione del verbo inglese, ha la possibilità di segnalare il proprio grado di DC per la posizione nella frase; $b$ ) il verbo di modo finito italiano supera il verbo inglese per il valore comunicativo lì dove, con le categorie di persona e numero, che rappresentano l'EPN, funge da tema o anche, nel caso della presenza del soggetto sostantivale, ne fa parte, mentre il verbo inglese, che non riporta l'EPN, no; c) il verbo di modo finito italiano supera il verbo inglese per il valore comunicativo lì dove, con il sistema dei tempi, ha la possibilità di esprimere diversi significati temporali, soprattutto grazie alla distinzione formale tra il perfetto e l'imperfetto; d) una forte tendenza all'uso di predicati sintetici fa sì che il grado di DC della componente nozionale del verbo italiano sia più alto del grado di DC del verbo inglese.

\section{Bibliografia}

Benincà, Paola (1991), "Ordine normale e ordini marcati”, in: RenzI, Lorenzo SAlvi, Giampaolo (eds.) (1991), Grande grammatica italiana di consultazione, vol. I, Bologna: Mulino.

Firbas, Jan (1959), "Thoughts on the communicative function of the English verb", Brno Studies in English, 1, 39-68.

Firbas, Jan (1961), "On the communicative value of the modern English finite verb", Brno Studies in English, 3, 79-98.

Firbas, Jan (1966), "Non-thematic subjects in contemporary English", Travaux Linguistique de Prague, 2, 239-256.

Firbas, Jan (1979), "A functional view of 'ordo naturalis'”, Brno Studies in English, 13, 29-59.

Firbas, Jan (1991), “Dagli studi comparativi sull'ordine delle parole”, in: Sornicola, Rosanna - Svoboda, Aleš (eds.) (1991), Il campo di tensione, Napoli: Liguori, 347369.

FIRBAS, Jan (1992), Functional sentence perspective in written and spoken communication, Cambridge: Cambridge University Press.

KLímová, Eva (2002a), “Dislocazione a sinistra - descrizione formale e funzionale”, Études romanes de Brno, L 23, 71-79.

KLímová, Eva (2002b), “Ordinamento lineare dell'enunciato in italiano", Linguistica Pragensia, XII/1, 40-49.

KLíMOVÁ, Eva (2003), Italské určité sloveso z pohledu funkční větnéperspektivy ve srovnání s určitým slovesem anglickým, Opava: Slezská univerzita.

KLímová, Eva (2004), "Verbo italiano ed il verbo inglese sulle scale semantiche", Linguistica Pragensia, XIV/1, 32-41. 
Il verbo italiano di modo finito nella struttura informativa dell'enunciato...

KLímovÁ, Eva (2007a), "Il verbo italiano a modo finito ed il verbo inglese nel loro valore comunicativo", Linguistica Pragensia, XVII/1, 46-55.

KLímovÁ, Eva (2007b), "Fattori della prospettiva funzionale dell'enunciato in italiano a confronto dell'inglese e del ceco", in: KLíMOvÁ, Eva (ed.), In onore di Ivan Seidl. Opava: Slezská univerzita, 113-123.

KLímovÁ, Eva (2007c), “Osservazioni sulle scale semantiche in italiano a confronto dell'inglese e del ceco", Écho des études romanes, III/1-2, 173-181.

Mathesius, Vilém (1942), "Ze srovnávacích studií slovosledných", Časopis pro moderní filologii, 28, 181-190.

Sornicola, Rosanna - Svoboda, Aleš (eds.) (1991), Il campo di tensione, Napoli: Liguori.

Svoboda, Aleš (1989), Kapitoly z funkční syntaxe, Praha.

Svoboda, Aleš (1991), "Le posizioni nell'ordine delle parole ceco dal punto di vista dell'articolazione attuale“, in: Sornicola, Rosanna - Svoboda, Aleš (eds.) (1991), Il campo di tensione, Napoli: Liguori, 423-452.

\section{Fonti dello spoglio}

JoyCE, James (1992), Dubliners, London: Penguin Books (Abbr. DUB).

Joyce, James (1995), Gente di Dublino (traduzione Marina Emo Capodilista), Luigi Reverdito Editore (Abbr. GEN).

Moravia, Alberto (1992), Gli indifferenti, Milano: Tascabili Bompiani (Abbr. IND).

Moravia, Alberto (1975), The Time of Indifference (traduzione Angus Davidson), Panther, Frogmore (Abbr. TIM).

Tomasi Di Lampedusa, Giuseppe (19937), Il Gattopardo, Milano: Feltrinelli (Abbr. GATT).

Tomasi Di LAMPEDUSA, Giuseppe (1996), The Leopard (traduzione di Archibald Colquhoun), London: The Harvill Press (Abbr. LEOP).

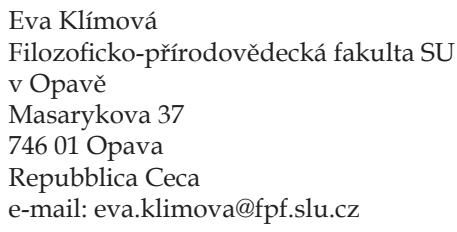

